ÉGYPTE monde arabe

\section{Égypte/Monde arabe}

30-31 | 1997

Les visions de l'Occident dans le monde arabe

\title{
Partir, ou la proximité entre « ici » et « là »
}

Émigrations imaginaires depuis trois villes marocaines

\section{Susan Ossman}

\section{(2) OpenEdition}

\section{Journals}

Édition électronique

URL : https://journals.openedition.org/ema/1608

DOI : $10.4000 /$ ema. 1608

ISSN : 2090-7273

Éditeur

CEDEJ - Centre d'études et de documentation économiques juridiques et sociales

Édition imprimée

Date de publication : 30 septembre 1997

Pagination : 145-158

ISSN : 1110-5097

Référence électronique

Susan Ossman, «Partir, ou la proximité entre « ici » et « là » », Égypte/Monde arabe [En ligne], 30-31 | 1997, mis en ligne le 08 juillet 2008, consulté le 07 juillet 2022. URL : http://journals.openedition.org/ ema/1608; DOI : https://doi.org/10.4000/ema.1608

Ce document a été généré automatiquement le 7 juillet 2022

Tous droits réservés 


\title{
Partir, ou la proximité entre « ici » et « là »
}

\author{
Émigrations imaginaires depuis trois villes marocaines
}

\section{Susan Ossman}

1 Malgré les contrôles de plus en plus stricts imposés aux flux migratoires à travers le monde, nombreux sont ceux qui, au Maroc, rêvent encore de partir à l'étranger pour y travailler ou y étudier. En 1992, nous avons demandé à un certain nombre d'habitants de Casablanca, de Tanger ou de Fqih Ben Saleh, ayant exprimé une volonté d'émigrer à un moment donné de leur vie, de bien vouloir répondre à nos questions concernant leur perception des « ailleurs » où ils espéraient se rendre.

2 Le terme d'«émigrant » est souvent associé à l'idée d'une personne ayant quitté son pays en raison de ressources économiques limitées. À la lecture de journaux européens ou américains, en discutant avec des fonctionnaires de consulats en poste dans des pays en voie de développement, ou en interrogeant les gens en Europe sur les raisons qui poussent les individus à émigrer, il ressort comme un « fait » évident que les étrangers résidant à l'extérieur de "notre » pays veulent y « entrer » afin d'améliorer leur sort. Les images de l'Europe ou de l'Amérique occupent ici une place prépondérante dans la mesure où elles semblent jouer un rôle important dans l'« exhibition de richesses " devant les plus démunis aiguisant ainsi leur appétit pour tout ce qui est « occidental ».

Pourtant, ce qui frappe le plus dans la façon dont les gens de tous âges et des deux sexes parlent de leur projet ou de leur rêve d'émigrer, c'est la fluidité et la récurrence des images oscillant entre l'«ici » et le «là-bas ». Ces façons d'exprimer leurs rêves ne peuvent être comprises à moins de renoncer à percevoir l'émigration comme un mouvement aberrant à travers des frontières politiques ou culturelles parfaitement délimitées. C'est en mettant en évidence les liens entre les manières dont les gens parlent de leurs rêves et de leurs projets et les transformations spatiotemporelles remarquables que connaît le monde actuel que l'on peut amorcer une réflexion sur le mode de création des " ailleurs » spécifiques. Contempler la gamme des possibilités de déplacement nous oblige à porter un regard plus large sur la circulation des individus, 
des objets et des images qui peuplent le monde et qui établissent ou rétablissent des régions (Foster, 1991).

Tout comme les touristes choisissent des modes de déplacement mettant en scène des lieux nouveaux depuis le point de vue des géographies fondamentales et des positions sociales - qu'ils déplacent avec eux -, les gens qui projettent d'émigrer conçoivent eux aussi l'« ailleurs » par rapport à l'« ici ». Ainsi, plutôt que d'opposer deux lieux ou deux images fixes de lieu, l'appréhension du phénomène migratoire exige que l'on s'attache au processus de formation des manières à partir desquelles les projets de vie et les déplacements à travers des espaces sociaux et symboliques sont envisagés. Les manières de fabriquer des images, de relater des récits et de se rapporter au déplacement sont imbriquées. Il importe de voir comment les modèles et éléments variés s'offrent aux différents individus et groupes ici et là. En outre, il faut garder à l'esprit que les images de Tailleurs, comme toute image, doivent être perçues non pas comme de simples reflets mais bien comme des éléments qui participent à la construction d'univers sociaux.

5 Les images modernes, les moyens de transport et les systèmes économiques, malgré leur rôle dans la diffusion de concepts tels que "tradition » et "authenticité », sont souvent perçus comme des facteurs de fragmentation de ces «totalités » qui lient l'expérience aux valeurs partagées. Cet éclatement apparent du tissu des relations sociales individuelles a souvent aussi été associé à l'impossibilité manifeste de construire des récits convaincants sur le passé et le présent en termes de choix sociaux et personnels clairs. Cette " fragmentation » du sens est, la plupart du temps, perçue comme problématique par les observateurs dont la recherche se fait par le « haut ", à partir d'une position objective. Ceux-ci sont souvent les derniers à réaliser non seulement l'erreur qui consiste à croire qu'une telle position peut être atteinte, mais aussi dans quelle mesure les transformations qu'ils dénoncent doivent être maitrisées par les gens afin qu'ils puissent créer des modèles de mobilité qui donnent sens à leur vie.

\title{
Héros, histoire et images
}

\begin{abstract}
« Il y a tellement de personnages historiques qui sont importants à mes yeux. Je peux nommer Muhammad, le Prophète, et je dois ajouter que je ne mentionne pas son nom seulement dans un sens strictement religieux puisqu'il est aussi celui qui a révolutionné et fondamentalement transformé l'histoire de l'Arabie et qui a unifié la communauté arabe. Je pense aussi à Mao, Lénine, Karl Marx, Engels et Hegel. Ou à des philosophes comme Foucault ou Antonio Gramsci. Je pense à Ho Chi Minh, Nelson Mandela, Agostinho Neto, des noms que je garde de l'époque où j'étais écolier. Il me vient aussi à l'esprit d'autres personnalités, comme Abdelkrim Khattabi, Laroui, Abdelkebir Khattibi, et à la formation musicale Nas al-Ghiwan. ${ }^{\circ}$ » (Abdelkrim, 36 ans, Fqih Ben Saleh, Maroc, 1992)
\end{abstract}

6 La liste très fournie des personnages historiques favoris d'Abdelkrim est analogue à celle de plusieurs autres personnes que nous avons interviewées le Prophète Muhammad figurait en tête de liste dans nombre de citations. La chute du mur de Berlin en 1989 n'a vraisemblablement pas affecté l'attrait qu'exercent Lénine, Marx, Mao ou Ho Chi Minh. De même, la défaite de Saddam Hussein n'a pas terni son image aux yeux de nombre d'entre eux. Le nom du roi marocain Hassan II a été mentionné par près d'un tiers des interviewés. S'il est regrettable, le fait que la plupart des gens ait plutôt cité des personnages masculins ne fut pas une surprise. Mais ce qui fut peut-être 
le plus marquant dans les réponses recueillies, c'est qu'elles ne se limitèrent pas à un héros ou à une héroïne. Alors que notre question était posée au singulier, plutôt que la description d'une vie exemplaire ou de l'influence exercée par un personnage en particulier dans leur vie, la réponse comprenait plusieurs noms, où revenaient souvent Rambo, Hassan II, Abdelwahab, Saddam Hussein et Muhammad. Des listes de personnages bien connus apparaissaient, à la manière de photos ou de publicités collées les unes à côté des autres au mur d'une épicerie de quartier. Des collages verbaux et visuels sont ainsi créés, et à partir de ceux-ci, il nous semble possible de saisir des styles dans les discours plutôt que des trajectoires personnelles.

7 En effet, les discours que nous avons écoutés, enregistrés et ensuite retranscrits ne peuvent être analysés si nous les appréhendons à partir du point de vue d'une idéologie ou d'un projet linéaire « cohérents » (Tozy, 1992). Par exemple, parmi ses héros, Hamid cite Che Guevara alors que son projet de vie est d'aller en Italie y faire de l'argent pour ensuite retourner chez lui et monter une affaire. De son côté, Jamila vante les vertus du souverain marocain alors que son jugement sur l'effort du gouvernement en vue d'« aider l'homme de la rue » reste très critique. Que ce soit en parlant de lieux éloignés ou en se définissant eux-mêmes, les individus ont tendance à produire des récurrences dans leur style et à reproduire un certain modèle dans leurs descriptions et opinions ils n'ont cependant pas toujours une "ligne" de pensée ou un comportement clairs (Devreux, Hillman, 1995 Ossman, 1994). Quand Ahmed parle, il accorde plusieurs termes français ou italiens au pluriel de la langue arabe. Perçus du point de vue d'un langage «correct» ou d'une perspective «logique », ces exemples peuvent nous amener à considérer le discours de ces gens comme étant «faux», contradictoire, voire erroné. Cela peut être considéré comme le résultat d'une confusion ou d'une fragmentation face à des transformations sociales soudaines. Pourtant, même si certains de ces individus se trouvent en effet dans une situation difficile ou ont déjà vécu plusieurs déplacements, d'autres ont confortablement vécu dans leur quartier depuis leur enfance. Par ailleurs, même dans le contexte des sociétés les plus instituées, les contenus les plus hétérogènes emplissent les cadres photographiques et les écrans de télévision. Est-il aujourd'hui inconcevable de réviser nos jugements relatifs aux contradictions à l'oeuvre dans les arguments "rationnels", de sorte que l'on puisse entendre les histoires des gens et les nôtres mêmes?

8 La manière de parler de nombre de ces émigrants potentiels diffère peu des séquences de termes imagés employés dans les déclarations gouvernementales ou les publicités télévisées. Les mots s'assimilent à des images en ce qu'ils sont juxtaposés et interchangeables d'un point de vue formel. Leur représentation photographique ou filmée est un phénomène qui comprend désormais le monde entier, et ce au moment même où des groupes d'experts s'appliquent à intégrer leurs normes dans les interactions sociales quotidiennes et les structures de pouvoir. En théorie, l'abstraction de la caméra, tout comme celle de l'individu moderne, a toujours la possibilité d'inventer de nouveaux contenus. Une offre diversifiée répondrait alors à une demande intarissable à l'échelle globale. Les affiches d'acteurs ou de dirigeants politiques et leurs noms sont alors mobilisés comme un corpus d'adages populaires. Chaque nouveau lecteur peut les associer à son gré et selon des caprices et désirs qui résistent souvent à l'analyse. À l'instar des collages de personnages célèbres montés par nos interviewés, le but de cette pratique consiste moins à adhérer à un système de pensée cohérent qu'à élaborer un style. Ce style repose sur une accumulation et une juxtaposition d'éléments 
disponibles selon des agencements spécifiques, un peu à la manière d'une garde-robe de dandy.

9 Les propos d'Abdelkrim ne comportent pas d'images de personnages célèbres. Il prend soin par exemple de donner les raisons pour lesquelles le Prophète Muhammad vient en tête de sa liste. Son point de vue n'est pas «idéologique », non plus qu'un simple reflet de sa "culture ». Il reflète cependant la trajectoire personnelle de quelqu'un qui garde en mémoire les mouvements d'indépendance des années cinquante et soixante et qui affiche un penchant «intellectuel». Parmi les personnages types qui peuplent son monde, plusieurs ne sont pas mentionnés, tels les étoiles du cinéma ou les hommes politiques contemporains. Par rapport à ce qu'Abdelkrim exclut, peut-on savoir, par défaut, ce qu'il souhaite peut-être devenir Où les héros nous mènent-ils

\section{Ici, là et ailleurs}

Les « ailleurs » au Maroc n'apparaissent pas sur une carte géographique. En général, ils sont associés à l'Europe, au Canada, aux États-Unis ou, plus rarement, à l'Australie. Il va sans dire que les raisons pour lesquelles on en vient à "connaître " ces lieux sont multiples. Bien que beaucoup à Fqih Ben Saleh aient de la parenté et des amis établis en Italie, c'est l'Espagne qui demeure la destination favorite des habitants du nord du Maroc. Les Casablancais ont des destinations plus variées, mais les liens avec la France y sont peut-être plus évidents là et à Rabat, que dans les villes de Tanger et de Fqih Ben Saleh. Alors que l'Europe tend à être "connue " grâce aux récits personnels, aux médias ou à la littérature, l'Amérique et l'Australie sont plutôt connues par l'intermédiaire de films, d'ouvrages ou d'expériences spécifiques. En réponse à nos questions sur le racisme ou sur les difficultés qu'ils pourraient rencontrer "ailleurs ", les interviewés ont tendance à établir une hiérarchie, une sorte de "palmarès des dix premières destinations ». Certains des individus consultés font des interprétations élaborées des traits culturels spécifiques qui rendraient certaines destinations préférables à d'autres. Ainsi, quelques-uns soutiennent que l'Italie est plus "méditerranéenne », et qu'il est donc plus facile de s'y plaire qu'en Grande-Bretagne ou qu'en France. Alors que cette apparente "proximité » est perçue par certains comme un avantage, d'autres la conçoivent comme étant potentiellement problématique, $\mathrm{c} r$ ils considèrent leur situation actuelle comme résultant en partie de ce qu'ils appellent des caractéristiques culturelles «méditerranéennes ». Ces derniers ont tendance à opter pour l'« aventure » ou pour un « changement total » en Amérique ou en Australie. Dans tous les cas, plusieurs sources sont exploitées et citées de façon critique, en vue de créer des images de ces divers ailleurs. Les distances géographique et culturelle sont ainsi souvent confondues.

11 La carte (géographique) moderne peut être vue comme représentant un monde homogène. Les photographies, les images télévisées ou les journaux reproduisent cette division d'un espace circonscrit et contribuent à l'extraction d'images spécifiques de leur contexte, permettant ainsi leur diffusion à travers le monde. Pourtant, à l'intérieur de ces cadres, certains éléments sont identifiés comme étant «modernes» ou «traditionnels », « occidentaux » ou « locaux ». En délimitant le mouvement en termes de frontières nationales, « ici » et « là » finissent par épouser l'imagé de la nation, tout comme finit par le faire l'identité de ceux qui en font partie. La porosité variable de ces frontières a des conséquences de taille sur la façon dont les individus imaginent et 
relatent leur expérience d'ici et d'ailleurs. Et ce, même si ce n'est pas uniquement en termes d'identité nationale ou de " territoires » locaux qu'ils le font. Casablanca, Rabat, Fqih Ben Saleh ou Tanger peuvent en effet alimenter l'imagination des habitants d'autres régions du Maroc même. Tandis que plusieurs tiennent compte des « obstacles » que constituent l'obtention d'un visa et l'adaptation à un nouveau milieu, il arrive souvent que leur état de privation et le sentiment d'injustice qui en résulte soient perçus en termes moins nationaux.

Leïla, par exemple, tente de quitter le Maroc définitivement. Là, dit-elle, c'est son père qui règne. Elle ne souhaite aucunement avoir un mari qui le remplacera en tant que supérieur en droit. Pour elle, partir veut dire se rendre en un lieu où les lois sont égales pour l'homme et la femme. Cet ailleurs peut se trouver dans n'importe quel pays, insiste-t-elle. Tout ce qui compte, c'est que son passage d'ici à là implique un nouveau type de relations avec les hommes en général, et avec son père en particulier. Étant une jeune fille aisée, sa vie est remplie d'amis, d'objets et d'images qui donnent corps à cette volonté d'une égalité entre l'homme et la femme. Seul son père perturbe cette image d'un monde où le mouvement est libre et aisé. Elle ajoute qu'auparavant elle étudiait à Paris. «Là-bas, dit-elle, j'avais l'habitude d'aller danser dans les boîtes. Oh, comme j'aime danser Je ne portais aucun intérêt aux hommes dans ces boîtes. Tout ce que je voulais, c'était bouger au rythme de la musique. » Après son retour au Maroc pour les vacances, et quand son père lui refusa son retour en France, elle passa une année complète dans sa chambre. "J'avais tout il me donnait tout. Mais je ne voulais pas le reconnaître. Tout me rappelait à mon emprisonnement. Un oiseau dans une cage dorée.»

Leilla est littéralement " coincée " dans une situation où, plutôt que de se déplacer librement à travers la carte du monde, les choses, les gens et les idées lui sont présentés par un père qui, à l'image du roi du Maroc, lui propose un accès absolu à toute chose en mouvement, insistant seulement pour qu'elle reste sur place. Alors que tout évoque le choix, aucun choix sous aucune condition n'est offert. La situation de Leilla contraste tout à fait avec celles de plusieurs personnes que nous avons interrogées. Beaucoup, à l'instar de Toufik, espèrent quitter le Maroc pour l'«ailleurs ", à la recherche des choses mêmes qui servent d'alibis aux geôliers de Leïla. Lui, comme Samira, espère partir pour gagner de l'argent, «économiser un peu » et monter une petite entreprise au retour. Dans ce cas, personne en particulier ne semble empêcher leur départ. L'ailleurs apparaît comme un endroit où l'on acquiert des expériences qui auront des conséquences sur le statut « ici ». C'est en même temps un vaste champ d'expérience et un endroit qui permet de rêver. Il condense les notions de possibilité, de changement, et de mouvement.

14 L'« ailleurs » est à la fois ce qui donne accès à des champs de pensée et d'action pour l'«ici » et ce qui offre un point de comparaison. Mais dans la mesure où cet endroit n'est pas encore habité par soi, il est aussi celui de toutes les ouvertures, souvent liées au plaisir, mais jamais aux réalités du retour. Pour sa part, Farid remarque « Pour que mon projet marche, il faudra que je travaille dur. Tu vois tous ces types revenir d'Italie avec de belles voitures. Ils travaillent dur et ne dépensent pas un sou lors de leur séjour. » Certains des interviewés ont évoqué le racisme, les inégalités, soulignant ainsi que «tout n'est pas rose là-bas ». Mais en parlant du racisme, de la pauvreté et du chômage, ils mentionnaient quelques pays en particulier (France, Belgique, Italie, ÉtatsUnis, etc.), pour ensuite ajouter soit que ces problèmes étaient beaucoup moins 
présents dans un autre «lieu» moins connu, soit qu'ils s'inscrivaient dans une situation globale.

L'« ailleurs », bien qu'il soit souvent assimilé au Nord ou à l'Ouest, est moins un lieu précis qu'une relation à la production de savoir, de richesse et de succès. Des images qui sont offertes à un moment précis dans le temps changent souvent au gré de la thèse que l'on cherche à défendre. Au Maroc, l'accès aux images, aux ressources disponibles et au déplacement est important non seulement parce qu'il donne aux gens les moyens de voyager, mais aussi parce qu'il leur permet de créer des "modes" d'action conformes aux milieux inédits qu'ils vont découvrir. Souvent, l'image de Marocains faisant la queue toute la nuit à l'extérieur des ambassades étrangères, revient pour exprimer la difficulté du passage d'ici à là. Cette réalité de l'attente et ce sentiment de savoir comment "passer " et être «choisi " pour l'obtention d'un visa sont souvent vécus de façon étrange. Les personnes "sélectionnées " attribuent souvent leur sort à leur capacité de « parler la langue » de leurs interlocuteurs bureaucrates.

Les images des divers ailleurs changent en fonction des thèses en présence. Si Toufik est fier d'avoir un ami et cherche à accroître notre estime pour lui en présentant l'Italie comme un havre de culture et d'apprentissage, il n'en développe pas moins des images alternatives de ce pays en parlant du racisme qu'il y a vécu. Plutôt qu'à un signe de contradiction, la production de plusieurs versions d'un lieu ou d'une expérience est souvent associée à une connaissance approfondie de ceux-ci. La complexité des images de tout pays, de toute ville ou de toute région, provient d'une connaissance pratique de ce lieu qui peut être aussi bien individuelle que collective.

Le frère d'Abdellah vit à Rome et peut l'aider à s'y établir lorsqu'il aura obtenu un visa. Bien qu'il ait beaucoup entendu parler du Canada, de la France ou de l'Espagne à travers les médias, il soutient que le seul fait de connaître là-bas quelqu'un d'«ici » rend ce départ possible. Abdellah « sait » que l'Italie n'est pas un endroit facile " pour un Marocain ». On y trouve des « racistes » et le travail y est difficile. Pour Abdellah, cet endroit est moins une page blanche qu'un lieu déjà "connu» par procuration. La réduction de l'incertitude que sa relation avec son frère permet est déterminante dans ses choix. Il diffère en cela de Leïla, qui, pour sa part, perçoit le départ vers railleurs comme une manière de fuire les liens familiaux et patriarcaux.

18 Ceux dont le projet est de "s'échapper " vers, des lieux présentés comme absolument «autres » rappellent des schémas historiques d'émigration au Maroc. Dans ce pays, avant l'avènement des cartes d'identité et des routes, un hors-la-loi, un paria, ou encore une famille déracinée par la famine ou la sécheresse pouvaient se déplacer librement. Dans le passé, et encore aujourd'hui, ces déplacements se faisaient du sud vers le nord du pays. En fait, le sentiment d'être un paria ou inapte économiquement constitue un leitmotiv dans presque tous les entretiens que nous avons eus avec ceux qui cherchent à partir. Quand nous leur avons demandé comment ils se situaient par rapport aux autres Marocains, presque tous ont répondu qu'ils faisaient partie des " couches inférieures de la société ». Même ceux qui disaient appartenir à la «classe moyenne » soutenaient qu'au Maroc l'absence de mobilité sociale était une justification suffisante au départ. Beaucoup ont affirmé que si leur situation personnelle pouvait être améliorée, ils ne voudraient pas quitter leurs famille et amis. Par contre, selon plusieurs, cette amélioration n'est possible que si des changements majeurs se produisaient dans le pays. Ces derniers sont appréhendés en termes de modernisation et sous-tendent des relations différentes avec la bureaucratie d'État, l'école et les 
employeurs. Les façons d'exprimer ce changement relèvent du même mode de traitement d'« images » que lorsque les « ailleurs » occupent la place centrale dans les discussions. D'autres périodes et d'autres lieux sont souvent sollicités pour illustrer un temps ou un espace qui font défaut comme des promesses d'égalité démocratique évoquant le mouvement à la manière d'un tableau de Mondrian.

En outre, ce nest pas simplement un espace «moderne » et égalitaire que les individus recherchent. Et cela, parce qu'existe aussi le sentiment que cette « modernité » est ici inatteignable et, qu'en fait, si elle ne l'était pas, elle détruirait les qualités mêmes que les gens veulent préserver en quittant leur pays. Rachida soutient que le fait que «les Français ne se rassemblent plus en famille » est un avatar de la "modernité ». Mais en même temps, partir est pour elle un moyen de se défaire des liens familiaux qui l'étouffent. Où les images romantiques des héros révolutionnaires d'Abdelkrim trouveraient-elles place dans un tel monde L'idée d'un territoire ouvert se double de la notion d'«êtres pour un territoire». La dualité de la carte moderne implique la désignation de la terre et du pouvoir en tant que nation, et l'identification de l'individu à l'entité ainsi créée. Les récits sur le développement, la mobilité et l'« amélioration » imaginés par la plupart des interviewés ne sont pas dénués d'« histoire ». En effet, leurs projets participent à la construction d'un temps historique, même si ce n'est qu'à l'échelle de l'individu. Dans une certaine mesure, ils reflètent aussi une acceptation des divisions sociales entre ceux qui se déplacent (ou devraient pouvoir le faire) et ceux qui « devraient être maintenus en place » (Lenclud, 1992).

\section{Savoir et pouvoir}

En sortant du consulat d'Italie, Amal déclare «Je comprends pourquoi une personne sans éducation peut éprouver de la difficulté à obtenir un visa, mais pourquoi moi ». Hind, un professeur d'université, accompagne son boucher au consulat de France " pour l'aider».

"C'est parce que je sais qu'il doit vraiment voir son fils malade en France. Je connais des gens influents là-bas. Pour l'accompagner, je me suis assurée d'être bien vêtue. J'ai mis un beau chapeau. Quand nous sommes arrivés, j'ai dû "sortir" mon meilleur français pour corriger celui des Françaises qui nous ont accueillis. "

Elle affiche ainsi son propre pouvoir à travers sa capacité à " aider » Jamal. Supposant qu'il est incapable d'obtenir un visa sans elle, elle peut aussi se montrer « supérieure " à la secrétaire française. Sa revendication d'un statut plus élevé du fait de sa connaissance de l'« autre » lieu se retrouve dans le récit de Jamal. Il a passé quelques années en Italie et est fier de connaître plusieurs « intellectuels italiens ». Aujourd'hui, il lit et écrit couramment l'italien. Par contre, il est retourné au Maroc parce qu'en Italie il n'a jamais « terminé quoi que ce soit » dans la réalisation de ses rêves. Même s'il a dû affronter de nombreux problèmes là-bas, il se perçoit encore comme étant d'une certaine façon "au-dessus" de la plupart des autres hommes au chômage qui le retrouvent chaque jour au café. À la question relative à sa profession, Toufik répond dans un mélange d'arabe, d'italien et de français qu'il était professeur de lycée. Malgré ses compétences linguistiques et ses contacts en Europe, il a fait l'erreur de retourner au Maroc avant d'avoir terminé son doctorat ou d'avoir gagné un peu d'argent. Il ne travaille plus comme enseignant et n'a pas réussi à trouver un autre emploi.

"Mais je suis quelqu'un de doué et je peux bouger", affirme-t-il. "Pendant un certain temps, ajoute-t-il, j'ai cru à l'adage selon lequel « $^{\circ}$ le pays de chacun est 
l'exil et l'exil est un pays ${ }^{\circ} \gg$. Mais maintenant, je me sens tout simplement en état

d'inertie. »

Le savoir rapporté d'ailleurs et le savoir sur railleurs peuvent être une source de pouvoir local, voire même un moyen d'accroître sa capacité à se déplacer. Au Maroc, les médecins comptent avec la légitimité de la médecine internationale. Les professeurs d'aérobic choisissent leurs clients parmi ceux qui sont le plus informés des dernières tendances internationales touchant leur domaine de spécialisation. On demande souvent à ceux qui « sont allés là-bas » de confirmer les images vues à la télévision ou les informations lues dans les journaux portant sur ces lieux. On peut obtenir une connaissance de railleurs sans avoir à se déplacer. Les jeunes fréquentent les centres culturels de diverses ambassades et adoptent souvent ce qu'ils croient être la mode vestimentaire et le comportement des jeunes du pays choisi. L'ailleurs est « ici » dans le sens où ce qu'on en connaît est intégré aux habitudes quotidiennes. Mais pour Toufik et pour ceux qui partagent son état " d'inertie », cette connaissance se présente de façon telle qu'elle sert plus à les aliéner qu'à leur permettre d'éprouver un sentiment réel de mobilité personnelle.

\section{Inertie, différence et sauts dans le temps}

La connaissance et le fait de se déplacer d'un lieu à un autre n'ont pas une valeur en soi. En revanche, ce qui importe est de savoir comment un changement de lieu peut transformer (ou être perçu comme transformant) des rapports à la société et à des ordres dominants d'espace et de temps. Les migrations, les flux d'objets et d'images se conjuguent pour donner naissance à des formations sociales spécifiques et modeler des interactions. Au moment où les scénarios habituels de mouvement progressif ne sont plus possibles, des relations alternatives au récit sont convoquées. Alors, les actions peuvent adopter les caractéristiques des hommes célèbres qu'Abdelkrim évoque si facilement les mouvements sont conçus de façon globale et instantanée. Un jeune de Fqih Ben Saleh raconte à Mustapha, sur le mode onirique, un projet d'émigration en ces termes :

« Là-bas (en Italie), tu peux même voler. Je peux m'asseoir dans un restaurant et, au moment où éclate une bagarre et où personne ne me regarde, je peux ramasser les vestes qui sont tombées des chaises et partir sans me faire remarquer. »

Dans ces propos, il n'y a aucune notion de projet, mais seulement l'idée de passer inaperçu pour profiter de ce qui peut «tomber par terre » à l'insu de tous. Tel les émigrants illégaux qui tentent de traverser le détroit de Gibraltar dans de petite barques ou à la nage, il n'est pas question d'affronter la réalité de façon directe. Les angles morts de la bureaucratie, mais aussi ceux d'individus inattentifs sont à exploiter comme dernier recours à l'action. Mais comment peut-on déceler de tels mouvements ${ }^{1}$ ?

Rappeler comment même ceux qui nourrissent des projets précis d'émigration débitent des listes de personnages historiques afin d'expliquer les liens entre ces histoires particulières et un mouvement migratoire plus vaste à travers le temps, constitue peutêtre une façon de mieux «situer " ces récits. Ces derniers peuvent être interprétés comme un mode de liaison logique du personnel au social pour ceux dont la marge de liberté de mouvement est si restreinte qu'il leur est impossible de rêver à l'échelle des territoires de la cartographie moderne. Suivant la logique de l'image proposée par les 
médias, qui, par ailleurs, participent aussi à la création de nouveaux modes disciplinaires dans les sociétés modernes, i'illisibilité soudaine de ces actes participe d'un monde où le "terrorisme " et la violence gratuite représentent des dilemmes majeurs.

Alors que dans chaque récit, l'obsession de Tailleurs peut être perçue comme l'indication d'un manque d'engagement au niveau «local» ou comme le reflet de l'attrait des "visions", diffusées par les médias modernes, d'un Nord plus prospère, elle semble inviter davantage à prendre en compte un rapport plus général au territoire, à l'espace et au temps. Les visions de Tailleurs ou de l'inaccompli sont liées à la fois à des ambitions présentes et aux cadres qui leur donnent sens. Si le "contenu » de ceux-ci peut être modifié à travers les voyages, en revanche, tout comme la pellicule photographique qu'on expose à de nouvelles formes, la perspective qui génère les images peut difficilement être altérée. Les changements de "cadre " correspondent à un pouvoir d'acquisition accru de ces nouvelles « lunettes ", non pas à des « visions du monde ». Alors, la capacité ou la tendance d'une personne à se rendre « ailleurs » doit être mise en rapport avec la possibilité qu'elle â d'émigrer et d'offrir à d'autres l'opportunité de le faire, et le pouvoir qu'elle a de déplacer de l'argent, des objets ou de l'information. Ainsi doit-on s'intéresser aux types de participation eux-mêmes et non pas aux possibilités de participation locale.

Observer les types de mouvement en général plutôt que figer les processus de migration dans les termes limités d'«ici » et de "là-bas", proposés par ceux qui parlent d'aller ailleurs, c'est voir comment ces mouvements permettent aux individus d'élaborer des récits, de collecter des brins de sens que Ton place bout à bout ou côte à côte, comme le fait Abdeikrim avec ses hommes célèbres. Parfois, la rencontre d'un nouvel élément lors de ses déplacements peut offrir de nouvelles possibilités de construction narrative ou donner davantage de poids à des choses déjà présentes. Dans ce sens, Mao ou Ho Chi Minh ne sont pas de simples représentants d'«idéologies » et le récit d'Abdelkrim affiche plutôt un trop-plein qu'un vide de sens. Chaque image est un point d'arrêt dans l'ensemble des trajectoires de vie possibles. Chaque nom offre une occasion de réflexion sur la variété de vies possibles. Connaître ces possibilités et les assimiler à des visages et à des noms ne revient pas à adhérer à une idéologie totalisante. Mais le fait même de commencer à envisager les choses à travers ces portraits sous-entend l'entrée dans un monde où la mobilité est possible. Détachées de leur contexte, aussi sûrement que le fut Toufik quand il « réalisa » que la neige d'Italie ne lui appartenait pas et que Dieu cherchait à l'en exclure, les images prennent de nouveaux sens au gré de leur réappropriation. Il est possible que les styles qu'elles illustrent soient incapables de permettre le " passage » d'ici à n'importe où. Par contre, l'accumulation de ces styles peut s'avérer essentielle pour déterminer la portée et la nature d'un scénario personnel qui, s'il veut connaître le "succès ", doit se déplacer vers les lieux les plus associés aux «ailleurs » individuels. À Casablanca, à Fqih Ben Saleh ou à Tanger, des pays ou des villes en particulier peuvent servir à représenter ces points dans le temps et dans l'espace. Mais, plus fondamentalement, ce sont les manières dont ces images peuvent varier et être réagencées qui permettent aux individus de se déplacer à l'intérieur de cercles plus ou moins restreints.

Les modes de connaissance, le sexe et le statut juridique et économique pèsent lourd dans la détermination à la fois du mode de différenciation des images de Tailleurs, mais aussi des manières dont les gens imaginent et mettent à exécution leur projet 
d'émigration. La qualité des liens aux images, aux objets ou aux idées disponibles à l'échelle mondiale ou régionale est déterminante dans la délimitation des choix (Eco, 1990). Les relations aux images, aux individus et aux projets ne sont ni infinies, ni globalement instituées. Leurs différences sont primordiales pour donner sens à un lieu et pour envisager ce que quitter ce dernier implique.

\section{BIBLIOGRAPHIE}

DEVREUX L, HILLMAN R., (eds), 1995, Fields of Vision. Essays in Film Studies, Visual Anthropology and Photography, Berkeley, Los Angeles, University of California Press (voir notamment « The Modernist Sensibility in Recent Ethnographic Writing and the Cinematic Metaphor of Montage »).

ECO U., 1990, The Limits of Interpretation, Indianapolis, Indiana University Press.

FOSTER R., 1991, « Making National Cultures in the Global Ecumene », Annual Review of Anthropology, vol. 20.

JACQUEMET M., 1992, « Namechasers, How Belonging to a Community Became a Crime », American Ethnologist, 19 (4).

LENCLUD G., 1992, « Le grand partage ou la tradition ethnologique » dans ALTHABE G et al., Vers une ethnologie du présent, Paris, éditions de la Maison des Sciences de l'Homme.

OSSMAN S., 1994, Picturing Casablanca. Portraits of Power in a Modem City, Berkeley, Los Angeles, University of California Press.

TOZY M., 1992, « Orient et Occident dans l'imaginaire d'un babouchier de Fez essai de lecture sur un mur d'images », dans BASFAO K. et HENRY J.-R., Le Maghreb, l'Europe et la France, Paris, CNRS.

\section{NOTES}

1. Nous pouvons comparer ces mouvements à ceux décrits par Marco Jacquemet (1992, p. 734) au sujet des actions de bandes de jeunes de banlieue en Italie méridionale. Contrairement aux organisations criminelles d'État ou d'autres plus anciennes comme la Camorra, leurs tactiques sont « intraitables».

\section{INDEX}

Mots-clés : émigration, identité, Maroc, Occident 
AUTEUR

SUSAN OSSMAN

CNRS/CELSA 